\title{
Critical Analysis of Salvage Radical Prostatectomy in the Management of Radioresistant Prostate Cancer
}

\author{
Daniel Seabra, Eliney Faria, Breno Dauster, Gunther Rodrigues, Gilberto Fava
}

Section of Urology, Pio XII Foundation, Barretos, Sao Paulo, Brazil

\begin{abstract}
Purpose: To critically evaluate salvage radical prostatectomy (SRP) in the treatment of patients with recurrent prostate cancer $(\mathrm{PCa})$.

Materials and Methods: From January 2005 to June 2007, we assessed patients with recurrent localized PCa. Recurrence was suspected when there were three or more successive increases in prostate specific antigen (PSA) after nadir. After the routine imagery examinations, and once localized PCa was confirmed, patients were offered SRP. Following surgery, we evaluated bleeding, rectal injury, urinary incontinence or obstruction and impotence. PSA values were measured at 1, 3, 6 , months and thereafter twice a year.

Results: Forty-two patients underwent SRP. The average age was 61 years. Following radiotherapy, the mean PSA nadir was $1.5 \mathrm{ng} / \mathrm{mL}$ (0.57-5.5). The mean prostate specific antigen doubling time (PSA-DT) was 14 months (6-20). Prior to SRP, the mean PSA was $5.7 \mathrm{ng} / \mathrm{mL}$ (2.9-18). The pathologic staging was pT2a: 13\%; pT2b: 34\%; pT2c: 27\%; pT3a: 13\%; and pT3b: $13 \%$. Bleeding $>600 \mathrm{~mL}$ occurred in $14 \%$ of the cases; urethral stenosis in 50\%; and urinary incontinence (two or more pads/day) in $72 \%$. The mean follow-up post-SRP ranged from 6 to 30 months. The PSA level rose in 9 , of which 6 had PSA-DT $<10$ months.

Conclusions: SRP is a feasible method in the management of localized radioresistant PCa. PSA-DT has shown to be important for the selection and SRP should not be performed if PSA-DT $>10$ months. Due to its increased morbidity, SRP should be only offered to the patients who are more concerned about survival rather than quality of life.
\end{abstract}

Key words: prostate cancer; radiotherapy; salvage therapy; prostatectomy

Int Braz J Urol. 2009; 35: 43-8

\section{INTRODUCTION}

According to estimates for 2008 from the Brazilian National Cancer Institute (INCA) 49,530 new cases of prostate cancer $(\mathrm{PCa})$ are expected in Brazil (1). Of these, more than 1,200 will be seen at our hospital. In the last decade, we have employed doses higher than 7,000 Gy in external beam radiation therapy (RT) to manage localized neoplasia. When localized recurrence is confirmed during follow-up of these patients, we recommend hormone blockade via androgen ablation or salvage radical prostatectomy (SRP). The first treatment method is considered palliative and the second is definitive with the intention to cure. Furthermore, when begun early in the course of recurrent disease, SRP allows excellent disease control without the need of concomitant hormonal treatment $(2,3)$.

Despite being employed for more than three decades, currently SRP is only offered to $25 \%$ of eligible patients who potentially are most likely to benefit from such therapy (4-6). The main reasons for 
this low rate of use are the technical challenges during the procedure and the increased risk of complications, such as urinary incontinence or obstruction, erectile dysfunction, and rectal lesions $(2,5)$. The aim of our study was to critically evaluate the role of radical prostatectomy in saving patients suffering from confirmed recurrence of prostate cancer restricted to the gland after treatment with conventional RT.

\section{MATERIALS AND METHODS}

In our medical service, between January 2005 and June 2007, we carried out a prospective study in which we evaluated patients in whom recurrent $\mathrm{PCa}$ was proven following external RT with more than $7,000 \mathrm{cGy}$ as early management with intention to cure for a localized cancer in clinical Stages I and II. Recurrence was suspected when there were three or more successive marker rises after PSA nadir, a criterion of the American Society for Therapeutic Radiology and Oncology (ASTRO). In these men, recurrence was ruled out by employing digital rectal exam to verify prostate status; pelvic magnetic resonance to study prostate and regional nodes; abdominal ultrasound to assess abdominal metastases; bone scintigraphy to rule out bone involvement; and thoracic radiography to rule out mediastinal or pulmonary disease. In the absence of dissemination, we performed a transrectal ultrasound-guided prostate rebiopsy (TRUS) to confirm prostate cancer. In this study, we only included patients with localized cancer, independently of PSA value. We excluded all men with negative rebiopsy and locally advanced or metastatic disease. Once recurrent localized tumor had been confirmed, all patients were proposed SRP. The procedure was fully explained and those who accepted the surgery were asked to sign the written informed consent for radical prostatectomy, which was adapted to be suitable for SRP. Following prostatectomy, the patients were evaluated for major complications inherent to the method: transoperative bleeding measured by aspirated blood; rectal lesions; urinary incontinence measured by numbers of pads used per day, considering incontinent patient that required 2 or more pads daily; urinary flow obstruction and erectile dysfunction. In the subsequent follow-up in order to control for neoplasia, we measured PSA after 1,3, and 6 months, and thereafter twice a year. Additionally, we calculated the PSA doubling time (PSA-DT) before SRP and correlated it with postoperative outcomes. We preferred do not perform survival studies since the median follow-up time is very short.

\section{RESULTS}

A total of 42 patients underwent SRP. The RT dose in 38 of these patients was 7,020 cGy, and 7,200 cGy in the remaining 4 . The mean age was 61 years old (59-69). The mean PSA was 9.2 ng/mL (4.5-39.0). Mean post-RT PSA nadir was 1.5 (0.57-5.5). Mean time to achieve nadir was 12 months (5-24). Mean PSA-DT time was 14 months (6-20). Mean pre-SRP PSA was 5.7 ng/mL (2.9-18) (Table-1). Pre-RT clinical staging (TNM) was as follows: T1c: $27 \%$; T2a: 27\%; T2b: 37\%; and T2c: 9\%. Post-surgery pathological staging (pTNM) was as follows: pT2a: 13\%; pT2b: $34 \%$; pT2c: $27 \%$; pT3a: $13 \%$; and pT3b: $13 \%$ (Table-2). Pre-RT biopsy Gleason histological grading was $5(3+2): 40 \% ; 6(3+3): 33 \% ; 7(4+3): 20 \%$; and 8 (4+4): $7 \%$. Post-SRP Gleason score was $5(3+2)$ : 20\%; $6(3+3): 20 \%$; $7(4+3): 46 \%$; and $8(4+4): 14 \%$. SRP mean time was 80 minutes (50-160). Dissection of the seminal vesicles was our most difficult step. Table-3 lists the main complications. Median blood loss was $300 \mathrm{~mL}$ and bleeding greater than $600 \mathrm{~mL}$ occurred in $14 \%$ of the cases. Urinary flow obstruction by urethral stenosis or bladder neck sclerosis occurred in 21 patients $(50 \%)$. These patients were submitted to internal urethrotomy and $5(12 \%)$ of them had pro-

Table 1 - PSA characteristics in 42 patients submitted to salvage radical prostatectomy $(S R P)$.

\begin{tabular}{lc}
\hline PSA Characteristics & Values (range) \\
\hline Pre-RT PSA (ng/mL) & $9.2(4.5-39.0)$ \\
PSA nadir (ng/mL) & $1.5(0.57-5.5)$ \\
Time to PSA nadir (months) & $12(5-24)$ \\
PSA - DT (months) & $14(6-20)$ \\
Pre SRP PSA (ng/mL) & $5.7(2.9-18)$ \\
\hline
\end{tabular}


Table 2 - Pre-RT and post SRP staging (TNM - 2002) in 42 patients who underwent salvage radical prostatectomy (SRP).

\begin{tabular}{cc}
\hline Stage & $\mathbf{\%}$ \\
\hline Pre-RT (clinical) & \\
T1c & 27 \\
T2a & 27 \\
T2b & 37 \\
T2c & 9 \\
Post SRP (pathological) & \\
T2a & 13 \\
T2b & 34 \\
T2c & 27 \\
T3a & 13 \\
T3b & 13 \\
\hline
\end{tabular}

longed obstruction signs requiring further endoscopic surgery. A third additional urethrotomy was performed in 2 patients. At the end of the study, all 21 patients were stenosis free. In $72 \%$ of the cases, incontinent patients required two or more pads daily. Erectile dysfunction occurred in $74 \%$ of the cases. Two rectovesical fistulas developed. One was a high fistula in the supratrigonal area, which occurred 32 days after surgery. The cause was probably inflammatory through adherence of the sigmoid colon to the bladder apex. This patient was subsequently treated using segmental colectomy, colostomy, repair of vesical lesion and late reconstruction of intestinal transit. The other

Table 3 - Main complications in 42 patients submitted to salvage radical prostatectomy (SRP).

\begin{tabular}{lc}
\hline Complications & Values \\
\hline Blood loss (mL aspirated) & $300(50-1200)$ \\
Urinary flow obstruction & \\
1 urethrotomy & $50 \%$ \\
2 urethrotomies & $12 \%$ \\
3 urethrotomies & $7 \%$ \\
Incontinence ( $\geq 2$ pads/day) & $72 \%$ \\
Erectile dysfunction & $74 \%$ \\
Rectovesical fistulas & $4.8 \%$ \\
\hline
\end{tabular}

patient had a low fistula in the vesicourethral anastomosis region and the cause was probably ischemic. A successful late approach with simple suture through the anus was performed. Median postoperative follow-up time was 18 months (range 1 to 36 months). In the subsequent assessments, 9 patients $(24 \%)$ had rising PSA. Of these, six had PSA-DT $<10$ months. In the remaining patients, the levels remained under $0.2 \mathrm{ng} / \mathrm{mL}$.

\section{COMMENTS}

In 2006, nearly half of the new patients with localized PCa in the United States elected RT as primary treatment (6). This choice hinges mainly on the concern of these men with quality of life, which in principle should be better than that of those who undergo radical surgery (7).

Factors influencing the success of RT can be patient treatment specific (8). Patient-specific factors can be stratified into 3 risk groups for relapse: the low risk group $(\leq \mathrm{T} 2 \mathrm{a}$, Gleason score $\leq 6$ and PSA $\leq 10)$; the intermediary group (T2b, Gleason score of 7 and PSA $\leq 20)$; and the high risk group (T3-4, Gleason score $\geq 8$ and PSA $\geq 20$ ). The parameters related to treatment included the modality of RT used (namely conventional, three-dimensional or intensity modulated conformal), the escalation and the maximum permissible dose. These are independent predictive factor of success with a failure rate varying between $25 \%$ and $32 \%$ (9). Another important point in the RT outcomes is the fact that $93 \%$ of the failures occur at the apex, an area that can pose a greater management failure risk and is difficult to treat due to its location (6). Following therapy, patients are periodically monitored for at least 15 years, an interval considered today as a curative criterion. In this period, due to the apoptosis of prostatic cells induced by treatment, reduced PSA levels are observed until the nadir, or minimum, level is reached. Thereafter, if there are successive increases in the marker level, recurrent neoplasia is suspected, which can be local or distant. If recurrence is confirmed by post-RT follow-up, it can have a profound impact on the patient's quality of life and the news can even be worse than the initial information that the patient has cancer $(2,8)$. 
Currently there is no definitive predictor criterion of local recurrence after RT. There are more than 100 different types described, with prostate cancer biopsy standing out, which is mandatory after 18 to 24 months after the treatment. Other criteria are nadir; the time to achieve nadir; PSA doubling time (PSA-DT); the ASTRO criterion (three or more successive rises in PSA) and PSA nadir + 2 ("Houston" +2) (8).

The value of a positive biopsy after RT is controversial, with a false positive rate of $60 \%$ and a false negative rate of $20 \%$ (10). The "traditional" ideal PSA nadir value is $<0.5 \mathrm{ng} / \mathrm{mL}$. In clinical practice, when there is a local recurrence, the average PSA nadir is $1.1 \mathrm{ng} / \mathrm{mL}$, and if there is a systemic recurrence, the PSA nadir is $2.2 \mathrm{ng} / \mathrm{mL}$. On the other hand, $80 \%$ of the patients who have survived 10 years after radiation therapy have PSA values up to $1.0 \mathrm{ng} / \mathrm{mL}(10,11)$. Thus, this would discredit the nadir of $0.5 \mathrm{ng} / \mathrm{mL}$ as a cutoff point. The average PSA nadir of our patients after radiation therapy was $1.5 \mathrm{ng} / \mathrm{mL}$.

The time to reach the nadir is important to understand the course of PCa after RT. Patients who achieve long-term disease control take longer to reach PSA nadir, an average of 24 months, while those who experience local recurrence had an average time to PSA nadir of 17 months and those with distant metastases attain PSA nadir in roughly 12 months $(10,11)$.

In patients with local recurrence, PSA-DT is greater than 6 months and in those with systemic recurrence PSA-DT is less than 3 months (12). Moreover, if PSA-DT $<10$ months, the 7-year survival after radiation therapy is only $52 \%(13,14)$. The mean PSA-DT of our patients was 14 months.

According to ASTRO criterion, biochemical recurrence alone does not justify the beginning additional therapy, because it is not considered a clinical recurrence (15). It is suspected if there are three or more successive rises in PSA after the nadir. Despite its high specificity, there is a lack of sensitivity because patients with distant recurrence are also being included. In addition, by using the current ASTRO criterion, the relapse can be diagnosed late, up to five years after radiation therapy, which can minimize the chances of a definitive cure (16).

Patients with confirmed tumor recurrence, had individualized management consisting of obser- vation without early treatment, brachytherapy, SRP, hormonal blockade, or even therapies considered to be experimental, such as cryosurgery and radiofrequency therapy $(6,8)$. The goal of these experimental therapies is to cause maximum destruction of prostate tissue with minimal damage to critical surrounding structures such as the urethra, urinary sphincter and rectum. On the other hand, the preservation of these structures may result in incomplete management, since some regions of the prostate are closely adjacent or adherent to these structures (6).

The justification for employing some kind of treatment is that the time interval from PSA failure until the discovery of the metastases varies from 3 to 8 years, after which death from cancer generally occurs within the next 5 years (17). Thus, certain groups of patients, especially younger ones, would benefit from early institution of definitive treatment (16). For this purpose, SRP provides survival rates similar to those of primary radical prostatectomy (16). Open retropubic SRP has been employed for more that 30 years with intent to cure after RT failure (3). Because it is considered more difficult and contains a higher risk of complications than primary radical prostatectomy, it is only currently offered to $25 \%$ of eligible patients who potentially are most likely to benefit from such therapy (6). In contrast, it is a procedure that is being constantly improved and presently its complication rate is not as high as it was in the past (18). The "ideal" candidates for such a procedure are the same as those for primary radical surgery: those with life expectancy of 10 years or more, no co-morbidities, highly motivated, i.e., those who accept increased surgical morbidity, with pre-radiation PSA values $10 \mathrm{ng} / \mathrm{mL}$, preoperative PSA less than $10 \mathrm{ng} / \mathrm{mL}$, pre-radiation PSA-DT $>10$ months and with both pre-radiation and preoperative localized clinical stage (2).

The surgery is tactically and technically similar to primary radical retropubic prostatectomy $(5,16)$. Also, laparoscopic salvage radical prostatectomy has been reported as a surgical approach (19). Pelvic lymphadenectomy should be extended because an extensive lymphatic involvement has been found in $7 \%$ to $11 \%$ of the cases $(6,8)$. This surgical step is considered critical whereas there are adhesions between the bladder and iliac vessels reported in $27 \%$ of patients. Furthermore, it is difficult to dissect the 
apex of the prostate and the seminal vesicles as a result of vasculitis, fibrosis and obliterations resulting from radiation therapy (8).

Contrary to what has been reported in the literature, where the mean blood loss reported was $900 \mathrm{~mL}$, in our study blood loss superior to $600 \mathrm{~mL}$ occurred in only $14 \%$ of the patients. None of them required blood transfusion. There was a high rate of stenosis/sclerosis of the vesicourethral anastomosis: $50 \%$ in our series versus an average of $18 \%$ reported in the literature. The high obstruction rate can be explained by the decreased tissue vascularization that occurs after RT, with consequent healing difficulty (6). The urinary incontinence rate in our study, defined as two or mores pads required daily was $72 \%$, which is far above the $45 \%$ reported in the literature. Furthermore, we have not yet evaluate the response of the incontinent patients to urinary physiotherapy, to which all patients have been submitted, through Kegel exercises, biofeedback or electrostimulation. Nearly, $74 \%$ of the patients reported worse erectile function, but we did not evaluate how many patients already had erectile dysfunction prior to surgery, or their rate of improvement after the treatment was instituted. The intraoperative rectal injury rates were considered insignificant, since it occurred in only one case. The rate of rectal injury reported in the literature was between $0 \%$ and $19 \%(3-5,8)$.

PSA-DT calculation seemed important to us, since 6 out of 9 patients with persistent PSA rise following SRP had PSA-DT $<10$ months.

This series could be considered limited in time, but not as regards the number of cases: in less than three years we were able to treat 42 patients. This can be a reflection of our concern to offer optimal care as well as the certainty that SRP remains one of the treatment modalities most capable of providing a definitive cure in these cases (6).

Nevertheless, given the shorter follow-up, we cannot yet show the relapse-free survival rates. Another important point to be taken into consideration is that we still need to study the prostate cancer patient's real concern regarding the quality versus quantity of life dichotomy.

It was interesting to observe that in the eligible candidates to SRP, after minute considerations regarding the outcome, both related to the discovery of recurrence itself and to the possible consequences of surgical-associated morbidity, the greatest concern for each patient was survival, even if they would experience a worse quality of life.

\section{CONCLUSIONS}

We consider that salvage radical prostatectomy should be offered with intention to cure following failure of external beam radiation therapy as a treatment method of localized prostate neoplasia. SPR, despite being more technically challenging than the primary radical surgery, is feasible. PSA-DT calculation has proven to be important in these patients' selection. As a result of postoperative complications, surgery should only be offered to those considered to be "ideal" candidates who are self motivated

\section{CONFLICT OF INTEREST}

None declared.

\section{REFERENCES}

1. Estimativa 2008: incidência de câncer no Brasil. Rio de Janeiro, INCA. 2007; pp. 33-4. Disponível em: http://www.inca.gov.br/estimativa/2008/versaofinal. pdf

2. Sanderson KM, Penson DF, Cai J, Groshen S, Stein JP, Lieskovsky G, et al.: Salvage radical prostatectomy: quality of life outcomes and long-term oncological control of radiorecurrent prostate cancer. J Urol. 2006; 176: 2025-31; discussion 2031-2.

3. Mador DR, Huben RP, Wajsman Z, Pontes JE: Salvage surgery following radical radiotherapy for adenocarcinoma of the prostate. J Urol. 1985; 133: 58-60.

4. Ward JF, Sebo TJ, Blute ML, Zincke H: Salvage surgery for radiorecurrent prostate cancer: contemporary outcomes. J Urol. 2005; 173: 1156-60.

5. Dall'Oglio MF, Barreto F, Paranhos M, Nesrallah A, Nesrallah L, Srougi M: Salvage radical prostatectomy: an alternative treatment for local recurrence of radioresistant cancer. Int Braz J Urol. 2006; 32: 550-6.

6. Huang WC, Kuroiwa K, Serio AM, Bianco FJ Jr, Fine SW, Shayegan B, et al.: The anatomical and pathological characteristics of irradiated prostate cancers may 
influence the oncological efficacy of salvage ablative therapies. J Urol. 2007; 177: 1324-9.

7. Berry DL, Ellis WJ, Woods NF, Schwien C, Mullen $\mathrm{KH}$, Yang C: Treatment decision-making by men with localized prostate cancer: the influence of personal factors. Urol Oncol. 2003; 21: 93-100.

8. Touma NJ, Izawa JI, Chin JL: Current status of local salvage therapies following radiation failure for prostate cancer. J Urol. 2005; 173: 373-9.

9. Zelefsky MJ, Fuks Z, Leibel SA: Intensity-modulated radiation therapy for prostate cancer. Semin Radiat Oncol. 2002; 12: 229-37.

10. Crook J, Malone S, Perry G, Bahadur Y, Robertson S, Abdolell M: Postradiotherapy prostate biopsies: what do they really mean? Results for 498 patients. Int J Radiat Oncol Biol Phys. 2000; 48: 355-67.

11. Zietman AL, Tibbs MK, Dallow KC, Smith CT, Althausen AF, Zlotecki RA, et al.: Use of PSA nadir to predict subsequent biochemical outcome following external beam radiation therapy for T1-2 adenocarcinoma of the prostate. Radiother Oncol. 1996; 40: $159-62$.

12. Sartor CI, Strawderman MH, Lin XH, Kish KE, McLaughlin PW, Sandler HM: Rate of PSA rise predicts metastatic versus local recurrence after definitive radiotherapy. Int J Radiat Oncol Biol Phys. 1997; 38: 941-7.
13. Lee WR, Hanks GE, Hanlon A: Increasing prostatespecific antigen profile following definitive radiation therapy for localized prostate cancer: clinical observations. J Clin Oncol. 1997; 15: 230-8.

14. Lee AK, D'Amico AV: Utility of prostate-specific antigen kinetics in addition to clinical factors in the selection of patients for salvage local therapy. J Clin Oncol. 2005; 23: 8192-7.

15. [No authors listed] Consensus statement: guidelines for PSA following radiation therapy. American Society for Therapeutic Radiology and Oncology Consensus Panel. Int J Radiat Oncol Biol Phys. 1997; 37: 103541.

16. Stephenson AJ, Eastham JA: Role of salvage radical prostatectomy for recurrent prostate cancer after radiation therapy. J Clin Oncol. 2005; 23: 8198-203.

17. Pound CR, Partin AW, Eisenberger MA, Chan DW, Pearson JD, Walsh PC: Natural history of progression after PSA elevation following radical prostatectomy. JAMA. 1999; 281: 1591-7.

18. Vaidya A, Soloway MS: Salvage radical prostatectomy for radiorecurrent prostate cancer: morbidity revisited. J Urol. 2000; 164: 1998-2001.

19. Vallancien G, Gupta R, Cathelineau X, Baumert H, Rozet F: Initial results of salvage laparoscopic radical prostatectomy after radiation failure. J Urol. 2003; 170: 1838-40.

\section{Correspondence address:}

Dr. Daniel Seabra

Rua Brasil, 1500

Barretos, SP, 14783-180, Brazil

E-mail: daniel.seabra@terra.com.br 